\title{
A simple monochromatic spark discharge light source
}

\author{
O.H. Chin and C.S. Wong
}

Plasma Research Laboratory, Physics Department, University of Malaya, 59100 Kuala Lumpur, Malaysia

(Received 19 June 1989; accepted for publication 17 July 1989)

A compact spark discharge tube operated as a monochromatic light source at $587.5 \mathrm{~nm}$ is described. The device is powered by a small $25-\mathrm{kV}, 3300-\mathrm{pF}$ doorknob-type capacitor switched by an atmospheric air spark gap. The peak luminous intensity of the 587.5-nm line emission from the helium spark discharge operated at $23 \mathrm{kV}$ and $900 \mathrm{mbar}$ is estimated to be $2.1 \times 10^{3}$ Candela into $4 \pi$ radians.

Spark light illumination has been used in high-speed photography since the beginning of the century and various designs of the spark discharge light source have since been reported ${ }^{1}$ with spark duration ranging from nanoseconds to milliseconds. We report here a simple and easy to build spark discharge tube for operation as a monochromatic point light source.

The design and construction of the spark discharge tube is shown in Fig. 1. The sharp-tip solid cone anode is made of stainless steel which is screwed onto a brass rod connected

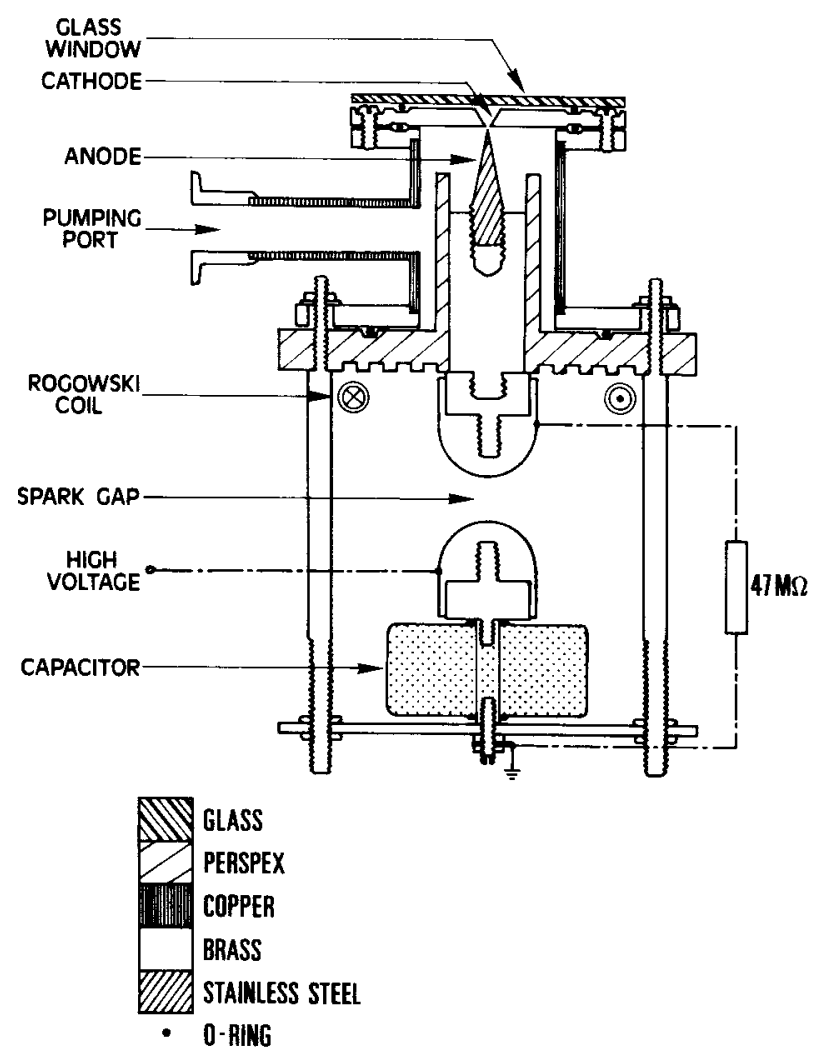

FIG. 1. Schematic diagram of the design of the spark discharge tube. directly to the spark gap switch. The cathode is a brass plate which has a tapered hole with a minimum diameter of $1 \mathrm{~mm}$ at its center above the anode. The vertical interelectrode spacing is set at $0 \mathrm{~mm}$. The cylindrical perspex sleeving surrounding the anode holder prevents arcing between the anode holder and the wall of the chamber. The return path from the cathode plate to the capacitor earth is via the chamber wall and six brass rods. A glass plate is placed above the cathode to seal the chamber at the required discharge pressure and to act as an output window.

The spark discharge tube is powered by a small doorknob type capacitor rated at $20 \mathrm{kV}, 3300 \mathrm{pF}$. However, the capacitor has been operated up to a maximum of $25 \mathrm{kV}$ with no apparent problem. The spark discharge is operated in the overvoltage mode and the switching is executed via a selffiring atmospheric air spark gap. A 47-M $\Omega$ resistor is used as a ballast resistor across the electrodes. The breakdown of this spark gap is set at $23 \mathrm{kV}$.

The spark discharge current is monitored by a Rogowski coil and the spark light output is detected by a BPX65 high-speed photodiode placed above the cathode hole.

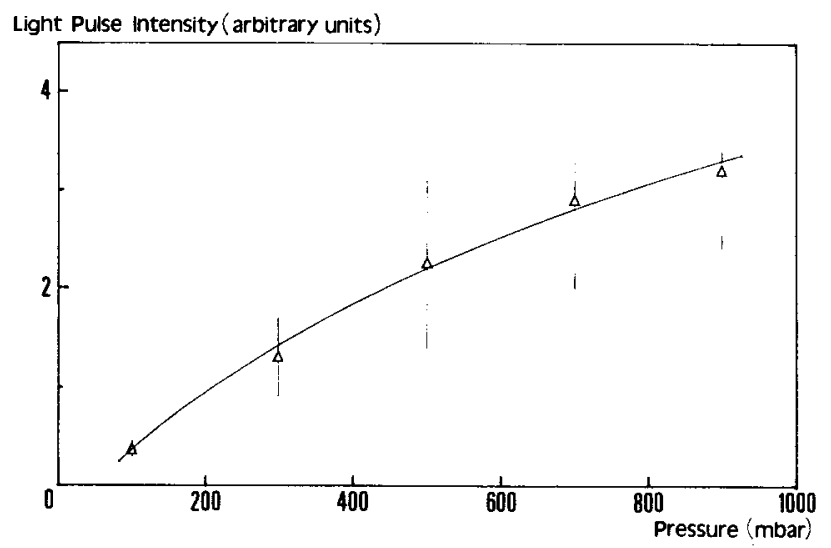

FIG. 2. Graph showing the relationship of the helium light pulse intensity with gas pressure. 


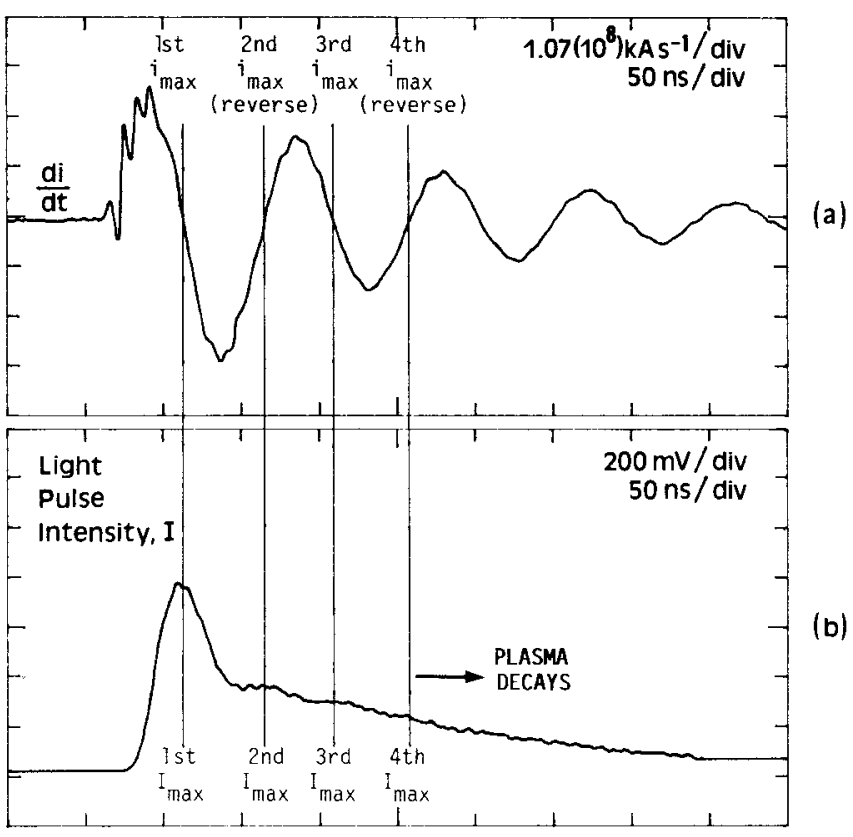

Fig. 3. Typical helium spark discharge light output at 900 mbar and its corresponding rate of change of current.

From the $d i / d t$ signal obtained, the first maximum of the discharge current $i_{0}$ is deduced to be approximately $5 \mathrm{kA}$ for a $23-\mathrm{kV}$ discharge. The spectrum of the light output is recorded onto Polaroid 667 film with the Chelsea Instrument Inc. Spectrograph S-100.

The spark discharge has been operated at different pressures of helium gas and found to give higher light output intensity at higher pressure. The relationship is shown in Fig. 2. A typical light output pulse obtained at 900 mbar of helium and its $d i / d t$ signal are shown in Fig. 3. It is observed that the light pulse begins to rise at the start of the current (first $d i / d t$ maximum ) to its maximum in a time of approximately $30 \mathrm{~ns}$, corresponding to the first maximum of the

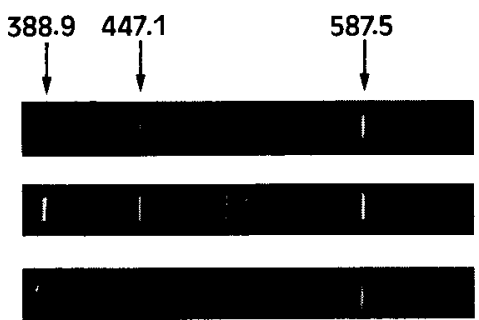

$\mathrm{nm}$

(b)

(c)

FIG. 4. Spectra obtained from Spectrograph S-100 for (a) helium spark discharge tube output; (b) standard helium spectral lamp; and (c) helium spark discharge tube output with Ealing bandpass interference filter at $587.5 \mathrm{~nm}$.

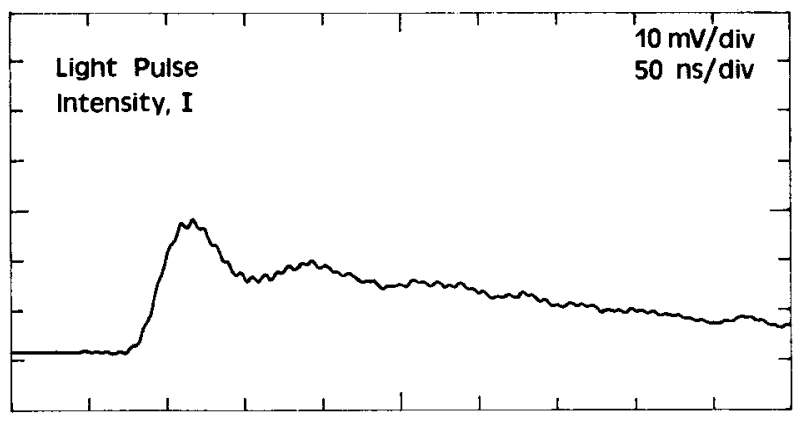

FIG. 5. The 587.5-nm spectral line output pulse at 900 mbar helium spark discharge.

current. Then the light intensity drops to half its maximum value in another $30 \mathrm{~ns}$ when the current has dropped to zero. Subsequently, the light intensity is observed to fluctuate with the current for the first two cycles of the discharge after which the plasma starts to decay. The spectrum of the spark obtained shows three prominent lines at $388.9 \mathrm{~nm}$ $\left(3^{3} P-2^{3} S\right), 447.1 \mathrm{~nm}\left(4^{3} D-2^{3} P\right)$, and $587.5 \mathrm{~nm}\left(3^{3} D-2^{3} P\right)$ as shown in Fig. 4(a). This spectrum is compared to that of a standard helium spectral lamp as shown in Fig. 4(b).

By introducing a bandpass filter with a window corresponding to one of the spectral lines of the discharge, a monochromatic light output can be obtained. As an example, the $587.5-\mathrm{nm}$ spectral line of helium is selected with an Ealing bandpass interference filter. The spectrum obtained is shown in Fig. 4(c), which shows a single spectral line at $587.5 \mathrm{~nm}$. This corresponding light pulse is shown in Fig. 5. From the specifications of the photodiode BPX-65 quoted by the manufacturer, the peak luminous intensity of the monochromatic spark discharge source at a discharge voltage of $23 \mathrm{kV}$ and helium gas pressure of $900 \mathrm{mbar}$ is estimated to be $2.1 \times 10^{3}$ Candela into $4 \pi$ radians.

The spark discharge tube can be operated with other gases to produce the corresponding spectra, and when combined with the appropriate filter, can be used to generate monochromatic light pulse of various wavelengths. The relatively long decay of the light pulse may be removed by the use of a critically damped discharge circuit. For the present system, a resistance of about $9 \Omega$ will be needed.

It is the intention of the authors to collimate the output light pulse of the above described spark discharge tube into a parallel beam using a system of lenses and to use it as a monochromatic light source for the optogalvanic study of a helium glow discharge.

This work is supported by the Government of Malaysia and the University of Malaya under the IRPA Program 4. 07-04-40.

${ }^{\text {'}}$ F. B. A. Früngel, High Speed Pulse Technology, Vol. II (Academic, New York, 1965), pp.1-214. 